\title{
Editorial
}

\section{Diagnostic utility of bone marrow sampling in HIV positive patients}

Bone marrow sampling is considered a useful investigation for the symptomatic patient with HIV but in view of the fact that it is an invasive (and uncomfortable) procedure how effective is it at providing the information required? The paper by Brook et al in this issue of the journal ( $p$ 117) attempts to provide some basic guidelines concerning the usefulness of bone marrow samples based on their experience of 215 patients with HIV or AIDS and 246 samples ( $87 \%$ trephine and aspirate) at a single London centre. All the patients had been investigated appropriately before bone marrow sampling was undertaken. Unfortunately, there are no details of the extent of the other investigations employed and it would have been useful to know whether or not the same system was in use both for the culture of bone marrow aspirates as well as for routine mycobacterial blood cultures.

Bearing in mind these limitations the largest and most informative group investigated were those with a pyrexia or fever of unknown origin (PUO or FUO). Some additional information is provided for cytopenic afebrile patients and those with a previously proved haematological malignancy. In the latter area the numbers are relatively small (38 samples). The authors considered bone marrow sampling to be an essential procedure for the cancer and therefore did not address the important question for patients and doctors of what additional information and benefit bone marrow sampling provided for these patients. Is complete staging of cancers really necessary in late stage HIV where the treatment goals for cancer may be very different from those in other situations? Perhaps we need some further work and guidelines on when staging is appropriate depending on the patient's suitability for aggressive therapy as assessed, for instance, by a low CD4 count, previous opportunistic infections and a lack of response to effective anti-HIV therapy.

Historically, the use of bone marrow samples for the investigation of difficult infections such as Salmonella typhi and Brucella species probably arose because of poor blood culture systems. However, by 1974 Christie noted that while bone marrow culture for Salmonella typhi might be successful when blood culture was not, he thought it "was doubtful if the procedure can often be essential for the diagnosis". ' Brook et al noted that of those with PUO, 33 $(27 \%)$ patients had an additional diagnosis made with microscopy and in 10 patients ( $8 \%$ ) the bone marrow sample was the only positive diagnostic test (equally split between infections and tumours). The results are similar to a recent but smaller series from the USA on the use of bone marrow aspiration in the investigation of a PUO in HIV. ${ }^{2}$ In this latter series the diagnostic yield of bone marrow aspirate was $32 \%$ although in $86 \%$ the diagnosis was arrived at by other means. ${ }^{2}$ In $52 \%$ of cases the diagnosis was made by a bone marrow aspirate as rapidly or sooner than other means but in only $14 \%$ of cases was the bone marrow sample the sole means of making the diagnosis. ${ }^{2}$ When considering just the diagnosis of mycobacterial infections, another London group reported that bone marrow sampling was the sole method of making the diagnosis in one third of cases despite the availability of modern blood culture techniques. ${ }^{3}$

Brook et al found that a positive result was more likely in advanced HIV disease as assessed by a low CD4 count (median 20 cells $\times 10^{6} / \mathrm{l}$ ), anaemia (mean $8.8 \mathrm{~g} / \mathrm{dl}$ ), or a recent (within the last 2 months) fall in the haemoglobin, white cell, or platelet count. The majority of the new diagnoses were, in fact, mycobacterial infections (mostly atypical) and the sensitivity of bone marrow sampling in this situation was $60-65 \%$. Twenty one per cent of the samples were positive for acid fast bacilli or granulomas compared with $0-15 \%$ in other series ${ }^{4}$ while in $6 \%$ the cause of the fever was found to be a lymphoma. The only other infection detected was toxoplasmosis (one case and 1\%). The sensitivity of bone marrow sampling might have been greater still in those with advanced disease. Unfortunately, an analysis of the sensitivity of bone marrow sampling by CD4 count, anaemia, or those with recent haematological changes was not presented. This seems to be an important point that might allow us to focus our investigations for the benefit of the patient.

With regard to the afebrile cytopenic patients, five (23\%) additional diagnoses were made in the investigation of pancytopenia, one $(3 \%)$ in the case of anaemia, and none for the investigation of leucopenia. Ninety five per cent of the samples taken for the investigation of thrombocytopenia were consistent with a diagnosis of HIV related thrombocytopenia.

The authors conclude that a bone marrow sample is a useful investigation in those being tested for a PUO but that it rarely provides additional benefit in the diagnosis of other haematological disorders such as thrombocytopenia or anaemia. Provided vitamin B12 and folate levels have been estimated and all marrow suppressive drugs discontinued then additional information is also provided in the case of the afebrile patient with continuing pancytopenia. The authors state confidently that marrow sampling is only rarely of use in diagnosing leucopenia or neutropenia. However, they have not provided any analysis of possible benefits for patients of a negative result (such as ruling out lymphoma) which might halt further investigations or allow a continuation of treatment. Equally, the diagnosis of HIV related marrow dysplasia would now indicate a need to increase antiviral chemotherapy rather than necessarily abandon therapy.

The summary guidelines will prove useful to others although it must be remembered that they are based on an essentially UK experience which may not be applicable even to other European countries. As an example, a recent article from Spain looking at PUO as a subject rather than the effectiveness of any particular test concluded that liver biopsy was the most effective investigation in nearly $70 \%$ of cases followed by bone marrow aspirate/biopsy in $40 \%$ of cases. ${ }^{5}$ Equally, in the investigation of thrombocytopenia ( $<100$ cells $\left.\times 10^{9} / 1\right)$, a bone marrow sample to exclude visceral leishmaniasis had a negative predictive value of $95 \% .^{5}$

The authors discuss the problems of morbidity and 
mortality. There is no doubt that a bone marrow aspirate is safer than a liver biopsy but we cannot assume a total lack of significant morbidity. Bone marrow aspiration may have a low morbidity from the professional point of view but it may not be low enough as far as the patients are concerned. If the same information can be obtained from other, less invasive investigations, then these should obviously be employed. The authors' suggestion that the technique should be considered after one month is a good one and supports the conclusion of Engels et al that bone marrow aspiration is indicated when a diagnosis is urgently required or when other methods have failed to provide a result. ${ }^{2}$ The advent of improved blood culture technology (as for Salmonella and Brucella) has rendered bone marrow sampling for the diagnosis mycobacterial disease less necessary and a direct comparison of the sensi- tivity and specificity of both techniques in the context of HIV would be welcome.

Regional Infectious Disease Unit, Consultant Physician and Reader

City Hospital and Department of Medicine,

Royal Infirmary, Edinburgh

1 Christie AB. Infectious diseases: epidemiology and clinical practice. 2nd ed. (\$) London: Churchill Livingstone, 1974.

2 Engels E, Marks PW, Kazanjian P. Usefulness of bone marrow examina tion in the evaluation of unexplained fevers in patients infected with human immunodeficiency virus. Clin Infect Dis 1995;21:427-8.

3 Riley UB, Crawford S, Barrett SP, Abdalla SH. Detection of mycobacteria in bone marrow biopsy specimens taken to investigate pyrexia of unknown origin. F Clin Path 1995;48:706-9.

4 Zon LI, Groopman JE. Hematological manifestations of the human immunodeficiency virus (HIV). Sem Hemat 1988;25:208-18.

5 Lozano F, Torre-Cisneros J, Bascunana A, Polo J, Viciana V, Garcia-? Ordóñez MA, et al. Prospective evaluation of fever of unknown origin iñ patients infected with human immunodeficiency virus. Eur $\mathcal{F}$ Clino Microbiol Infect Dis 1996;15:705-11. 